\title{
Nehuen Mapu Historia de una Relocalización Forzada
}

\author{
Juan Alonso Pacheco Rivas ${ }^{1}$
}

Trabajo recepcionado: abril 2007

Trabajo aceptado: julio 2007

\begin{abstract}
RESUMEN
El presente trabajo desarrolla dos líneas de investigación en temas de pobreza y derechos humanos en las comunidades del cajón del Río Queuco en el Alto Bio Bio, Chile. La primera es un estudio del caso de la relocalización de la comunidad de Nehuen Mapu en el fundo San Ramón en la comuna de Santa Bárbara desde la Comunidad de Malla Malla. La segunda es el contexto general de evaluación de la política indígena desde la perspectiva del derecho al desarrollo y los derechos humanos. En la primera línea se emplea la metodología cualitativa de investigación-acción, en la segunda la combinación de análisis de datos cualitativos y cuantitativos para los enfoques de evaluación. Los resultados obtenidos definen las causas y consecuencias de la relocalización de la comunidad y permiten postular un modelo de análisis y evaluación de la política indígena en Chile.
\end{abstract}

PALABRAS CLAVE: Pobreza, derechos humanos, políticas indígenas, relocalización.

\begin{abstract}
This work applied two research tracks in issues regarding to poverty and human rights at the communities of Bio Bio Highlands in the Queuco River valley in Chile. The first is a case study about the resettlement of the Nehuen Mapu community to the village of San Ramon, Santa Barbara County, from their previous Malla Malla Community. The second study regards the evaluation of indigenous policy from the perspective of development and human rights. In the first track we applied a methodology of action/research, whereas in the second track was applied a mixed Qualitative- Quantitative methodology in the evaluation. The outcome of this research shows the causes and consequences of the resettlement community Nehuen Mapu, and on the another hand we pursuit a model for an insight for evaluating of the current indigenous public policy in Chile.
\end{abstract}

KEY WORDS: poverty, human rights, indigenous policy, resettlement.

1 Sociólogo, consultor independiente, Investigador UNESCO Small Grant Programma en Temas de Pobreza y Derechos Humanos. juanpachec@gmail.com 
Revista CUHSO volumen 13 n $\div 1$

Introducción

La comprensión de los factores que determinan el desarrollo de los pueblos indígenas de Chile debe ser abierta a una reflexión permanente sobre el rol del Estado y los efectos que producen sus políticas y programas sociales, como también las consecuencias sociopolíticas de las tesis del Estado sobre la seguridad interior. El caso de las comunidades del Alto Bío Bío, en el contexto del conflicto histórico y la construcción de la represa Ralco, demuestra los efectos negativos de una intervención llevada adelante bajo paradigmas de modernización, de seguridad interior del Estado y desarrollo económico sin pertinencia cultural. En la actual relación del Estado de Chile con los pueblos indígenas, sus políticas y programas sociales, como es el Fondo de Tierras y Aguas Indígenas (FTAI), se detectan elementos que perturban a las comunidades y que generan una situación de profundización del conflicto histórico; como es posible de observar en este caso en las comunidades del Alto Bío Bío y en específico en la comunidad de Nehuen Mapu.

Los efectos culturales, así como los sociales y políticos, de la aplicación de programas sociales se traducen en una potenciación de los elementos ya presentes dentro del conflicto histórico de las comunidades mapuche, como lo es la violencia y la pobreza crónica de las comunidades. Los estados de violencia se expresan en una alteración al interior de las comunidades que impide su desarrollo, generando efectos en ellas que deterioran la calidad de vida y producen pérdida de potencialidades para la obtención de bienestar. Así también la violencia se traduce en una situación de pobreza crónica que encadena a un círculo que lleva a la disolución de las comunidades, pérdida de la cultura, empobrecimiento y, paradójicamente contrario a lo que el Estado de Chile se propone en su intervención, en conductas de las personas y comunidades que se oponen a la gobernabilidad política de parte de éste. Así se entiende que la intervención política del Estado de Chile bajo un prisma que no considera sus efectos en los factores señalados, y más aún opera bajo el criterio de "seguridad interior del Estado", produce un deterioro en el desarrollo y una crisis de gobernabilidad.

El caso que aquí se expone muestra claramente esta tesis, una política de intervención de un conflicto histórico que sólo lleva a extenderlo en diversas áreas del sector del alto y bajo Bío Bío, donde los grupos relocalizados expresan haber sido manipulados y abandonados por el Estado de Chile. Del mismo modo, el enfoque de las políticas públicas y sociales hacia los pueblos indígenas, en su manera fragmentada y parcializada de llevar adelante su intervención no visualiza las variables que determinan las relaciones conflictivas, replicando patrones de intervención que sólo las potencian y no se traducen en bienestar. Así puede indicarse que los factores determinantes que Ilevan a la situación de falta de desarrollo en las comunidades indígenas son: a) la pobreza crónica, b) la situación permanente de violencia, y c) la intervención política bajo el contexto de seguridad interior; transformando las expresiones del conflicto histórico de los pueblos indígenas con el Estado de Chile a nuevas expresiones del movimiento indígena que replican las situaciones señaladas. El cierre de este circuito es una permanente preocupación del Estado por asegurar su gobernabilidad a través de políticas que no tienen el impacto esperado, sino potencian el ya tenso conflicto.

\section{Metodología de Investigación}

El presente trabaj o se enmarca en dos líneas Ilevadas adelante por el autor dentro del proyecto de investigación para UNESCO. La primera de ella es la investigación participativa de Investigación/ Acción², en donde fue posible seguir una línea cualitativa de trabajo con el

2 Las fuentes de información directa corresponden a documentos de las peticiones, escritura de compras, fuentes de instituciones públicas y relatos directos de los involucrados. Las fuentes institucionales son el Municipio de Santa Bárbara, Municipio de Ralco, CONADI y Centro EULA de la Universidad de Concepción. 
fin de establecer la coherencia de las hipótesis causales que motivaron el fenómeno en estudio, la relocalización de la comunidad de Nehuen Mapu. La segunda línea de investigación se encuentra referida a la evaluación de la política indígena desde la perspectiva evaluativa del policy maker, estableciendo efectos y causas en las dinámicas sociales generadas por los programas de intervención en los problemas de pobreza crónica que se encuentran en aplicación a través de la política indígena, como lo es el "Programa Orígenes" y el Fondo de Tierras y Aguas Indígenas (FTAI) de CONADI. Desde ambas líneas se construye un estilo de evaluación de los efectos de la política indígena y la perspectiva de los derechos de los pueblos indígenas y los derechos humanos en el marco del "derecho al desarrollo".

Estas perspectivas de investigación se ubican dentro de una estrategia metodológica de combinación de enfoques y de herramientas cualitativas y cuantitativas como lo señala el grupo de investigación de la Universidad de Cornell, Q- Square (Howe, 2007). La combinación cualitativa y cuantitativa define así un método de trabajo que permite ir analizando un caso en específico que es el fenómeno de relocalización de la comunidad de Nehuen Mapu al sector de San RamónVillucura en la comuna de Santa Bárbara, como el contexto social y político en el cual este fenómeno se produce. Interesó los nexos necesarios entre el "estudio de caso" y las determinantes que llevan al proceso de relocalización a causa de tres fenómenos posibles de identificar: a) la escasez de tierra y extrema pobreza de la comunidad de Malla Malla, b) la intervención política de agentes externos en la comunidad de Malla Malla, y c) el clima de violencia permanente al interior de la comunidad como en su entorno social y geográfico.

En la perspectiva de análisis de desarrollo, en tanto, se pudo establecer un modelo de doce indicadores generales que establecen la relación entre la vulneración del territorio, los derechos de lospueblosindígenas, la alteración de las dinámicas internas de la comunidad y las acciones del Estado de Chile que las transgreden. Este modelo incluye cinco indicadores específicos que señalan los efectos en las dinámicas internas de la comunidad y otros siete indicadores que señalan a los elementos perturbadores provenientes desde las fuerzas externas a la comunidad. También fue posible identificar tres variables directas que afectan a la comunidad en términos de desarrollo y pobreza a las comunidades como efecto de la intervención directa desde los siete indicadores externos, además de una variable endógena en relación con los tres elementos señalados como variables de intervención externa. Se propone así un modelo analítico estructural que permite interpretar la acción del Estado y la sociedad chilena en las comunidades indígenas a través de la generación de pobreza por efecto de la ocupación histórica del territorio hasta la intervención del Estado de Chile por medio de los programas sociales y las políticas indígenas ${ }^{3}$.

Las variables identificadas corresponden a dos grupos de variables externas y dos grupos de variables endógenas:

El primer grupo externo de siete variables son:

1. Presión de tierras y conflictos de propiedad: situación generada por la acción de ocupación de sus tierras por parte de colonos y propietarios por acción fraudulenta, además del desplazamiento involuntario, persecución y hostigamiento producido en las campañas militares del Desierto y la Pacificación de la Araucanía.

2. Empobrecimiento material: existe una pobreza que está por debajo de la línea de la indigencia según datos de ficha CAS 2 (Municipio de Ralco, 2006).

3 Ver esquema final. 
3. Transformaciones de liderazgo: ruptura del liderazgo tradicional en un Lonko de guillatún y un Lonko político que ha generado divisiones al interior de las comunidades.

4. Asistencialismo, dependencia política y clientelismo: actitudes de parte de algunos comuneros y sus familias que ha favorecido el control social y generado nuevas redes de intercambio que intervienen en la dinámica tradicional de las comunidades, generando en ellas tensiones, conflictos y rupturas.

5. Violación de derechos humanos de primera generación: encarcelamiento, persecución y aplicación de la ley antiterrorista.

6. Exclusión social: La división de las comunidades tiende a generar grupos al interior de ellas excluidos de redes de cooperación, esto es producto del clientelismo.

7. Negación de identidad: en el caso de los evangélicos hay pérdida de identidad al no participar de los guillatunes, pero también hay pérdida de identidad en quienes tienden a participar de las redes asistenciales del municipio y del clientelismo del Estado.

Segundo grupo externo de cinco variables:

8. Ruptura generacional: hoy se han enfrentado al problema de la salida de jóvenes de sus comunidades a estudiar y han asumido nuevas formas de vida, discontinuando la memoria histórica y la ética transmitida por los ancianos.

9. Migración y desarraigo: el empobrecimiento y pérdida de identidad está generando la migración de familias y jóvenes fuera de las comunidades.

10. Alteraciones de la dinámica familiar: la migración de los jóvenes ha producido que ellos tiendan a cuestionar y criticar el sistema de vida tradicional y la transmisión de valores generacionales.

11. Alcoholismo, drogadicción y alteraciones mentales: se observa consumo en jóvenes y otros miembros de la comunidad de alcohol y otras drogas.

12. Alteraciones médicas, enfermedad y muerte prematura: se registran patologías endémicas y enfermedades crónicas en la población vulnerable.

Primer grupo de variables endógenas:

a) Escasez de recursos: corresponde al factor identificado como "presión de tierras", que se construye desde los indicadores señalados sobre tierras y recursos materiales, esta vez en forma cualitativa, si existen carencias que impiden la reproducción social y cultural.

b) Dependencia: se establece como indicador desde la información cualitativa registradas sobre las relaciones políticas de los dirigentes y líderes con el Estado en función de la construcción y toma de decisiones sobre los asuntos internos de las comunidades.

c) Pérdida de capacidades: existe una pérdida de conocimiento, capacidades y capital humano propio de la comunidad, que determina incapacidad de resolución de conflictos internos.

Segundo grupo de variable endógena:

d) Dinámicas internas: corresponde a la definición del espacio de vida comunitario desde criterios de seguridad y capacidades para construir riqueza. Es como la población se desenvuelve y considera pobre en el sentido de una forma de relación con la sociedad chilena o hacia su propia comunidad atribuyendo causas, razones y explicación a su situación de vida que abarca todos los espacios cognitivos, sociales y culturales.

Definido el contexto general de la investigación, es posible postular una relación entre la 
transgresión de los derechos humanos de las comunidades indígenas por la acción del Estado que lleva a la condición de pobreza crónica en razón a la aplicación de la política indígena. Sería posible, así, interpretar los resultados de la investigación del caso de la comunidad de Nehuen Mapu, en donde se refleja de manera directa los efectos de una política indígena aplicada con los criterios políticos clientelares y sin considerar los efectos señalados. Dicho de este modo, permite situar el caso dentro de un contexto permanente de violación y transgresión de derechos que afecta el desarrollo y determina un clima de pobreza y violencia. Estas últimas condiciones deterioran las posibilidades de bienestar de esta comunidad.

Los resultados integran la descripción cualitativa o cuantitativa, con evaluación de los efectos de las políticas públicas y sociales hacia los pueblos indígenas que se construyen sin los referentes políticos para su formulación, planificación, gestión y aplicación. Tales elementos de referencia lo constituyen el reconocimiento constitucional, la ratificación de nación multiétnica o pluriétnica, o el reconocimiento de convenios internacionales como lo es el Convenio 169 y el de Derechos de los Pueblos Indígenas. De esta manera el caso de la comunidad Nehuen Mapu demostraría los efectos negativos de los procesos de reasentamientos dentro del contexto del conflicto histórico causado por la intervención política, al aplicarse desconociendo el propio principio de derecho internacional articulado en la Ley Indígena vigente (art. 1).

\section{Resultados}

Descripción de la situación de la comunidad

La comunidad de Nehuen Mapu proviene de un grupo de catorce familias relocalizadas el año 2002 desde la comunidad de Malla Malla, las que son ubicadas en el fundo San Ramón- Michales, sumándose dos familias distintas a la comunidad de origen, una desde la comunidad de Trapa Trapa y otra de Ralco
Lepoy, totalizando 16 familias relocalizadas originalmente. El fundo San Ramón tiene una extensión de 160 ha, siendo principalmente de uso forestal, con baja tasación fiscal (grado 7 a 9 de tasación, según propias fuentes de CONADI en comunicación personal) y con una escasa disponibilidad del recurso agua, no posee riego, ni luz eléctrica, agua potable o algún tipo de servicio sanitario para las viviendas de los comuneros. Este fundo se encuentra rodeado, por un lado, de Bosques Arauco y Forestal Mininco, y por el otro del camino público; su acceso es un camino de tierra, solo ripiado en el comienzo, hasta hoy no posee demarcación de la subdivisión de la tierra según lo aplicado por CONADI en otros reasentamientos. Las viviendas de sus habitantes son mediaguas con cocina de fogón, sin ningún tipo de servicio básico, teniendo solo pozo negro y extracción de agua de una vertiente que en verano tiende a secarse. Las condiciones de vida son de extrema pobreza y lo of recido por CONADI en cuanto a posta de salud, sede social y escuela para los niños no se ha cumplido, teniendo beneficios otorgados por el Municipio dentro de las posibilidades legales que este puede, ya que al no estar regularizada la subdivisión, no reciben beneficios de vivienda o programas sociales, además que su acceso se encuentra restringido por estar fuera del $A D I$ 1, a la que originalmente pertenecían en la comunidad de Malla Malla.

Al momento de iniciar la investigación, los comuneros de Malla Malla se encontraban ya relocalizados en el sector de San RamónVillucura, existiendo un conflicto con las dos "familias externas" una de las cuales, se apropia de un sector de la casa patronal del fundo San Ramón y la otra estaba en permanente disputa con las 14 familias restantes. La situación de conflicto entre los miembros de la comunidad se produce por efecto de la división de la tierra hecho por CONADI, en la cual la asignación de predios a las familias se hace sin considerar su organización social básica ni sus relaciones sociales desde el territorio de origen. Se entregaron paños no mayores de 3,5 has, divididas entre sí en número de 3 a 4 por familia para totalizar 11,5 has, por 
familia ${ }^{4}$, un 10 a $20 \%$ del la cantidad de tierra original de Malla Malla que eran entre 80 y 120 ha ${ }^{5}$, generando conflictos de derechos de paso y uso por parte de los comuneros. Esto llevó a que la familia proveniente de Trapa Trapa fuera relocalizada en un predio individual, perdiendo así todo nexo con su comunidad de origen. Por otro lado, otras familias decidieron regresar a su comunidad de origen dejando abandonado sus predios y las familias que quedaron comenzaron a disputarse los escasos recursos madereros del fundo y a tener conflictos entre sí, también ante el riesgo de perder las veranadas desde donde se proveen de pasto para los animales y piñalerías para la recolección del piñón de araucaria.

El fundo San Ramón fue adquirido el año 2000 en 217 millones de pesos $^{6}$ a un particular de Santa Bárbara. Este predio fue comprado bajo la Ley Indígena 19.253 (artículo 20 b) en compra de tierras para solución de conflictos históricos, siendo este el que tenía la comunidad de Malla Malla ensus demandas frente a la escasa cantidad de tierras reconocidas en el Título de Merced original. Se adquieren 3.444 siendo 38.000 has, las demandadas históricamente, con un volumen de 15.000 has, superpuestas en 5.000 has, con la demanda histórica de la comunidad de Cauñicú sobre el mismo fundo, según datos aportados por Investigadores del Centro EULA. Así debe entenderse que esta relocalización se ubica dentro del histórico conflicto de la comunidad de Malla Malla con el Estado de Chile, y como tal es un efecto de la intervención de este por medio de sus políticas indígenas dentro de las lógicas de gobernabilidad y soberanía nacional.

La condición original de Nehuen Mapu desde la comunidad de origen que es Malla Malla es de extrema pobreza, como lo muestran datos de la ficha CAS 2 extraída desde el Municipio de Ralco. El puntaje de la comunidad es de 436 puntos, el que está por debajo de la línea de la pobreza indigente de la región, que es de 498 puntos, reflejando de manera directa el nivel de pobreza original de la comunidad. Los datos de la comunidad de Nehuen Mapu no fueron aportados por el Municipio de Santa Bárbara; sin embargo, se extrae desde los aportes de los funcionarios del Municipio que la condición de pobreza es similar o peor a la observada en la comunidad de origen ${ }^{7}$. Es así que en un principio los miembros de la comunidad aceptan ser trasladados desde la comunidad de origen frente a una oferta hecha por CONADI de mejorar su calidad de vida; sin embargo, en el proceso de investigación llama la atención la prontitud de aceptar lo ofrecido por CONADI en el año 2002 y a las precarias condiciones a las que fueron sometidos posteriormente. Puede observarse, al revisar los grupos de familias que fueron relocalizadas, un patrón común asociado a los roles ejercidos por ellos dentro de la comunidad de Malla Malla, siendo un grupo de familias de la línea de los Suárez Marihuan, cuyos representantes son Pedro Segundo Suárez Marihuan y José Martiniano Suárez Marihuan, y donde la beneficiaria de parte del terreno en este grupo era Agustina Huenupe Pavían de la comunidad de Cauñicú, esposa de Pedro, quien fallece el 13 de julio de 2002, y el otro grupo compuesto por la familia de Alberto Solar Huanque; ambos grupos familiares dirigentes de la comunidad de Malla Malla, tanto J osé Martiniano Suárez como Alberto Solar Huanque en calidad de Ioncos.

La condición de pobreza y abandono a la que posteriormente se ha visto sometida la comunidad de Nehuen Mapu ha llevado a los grupos familiares a un peregrinaje por el Municipio de Santa Bárbara, la gobernación provincial del Bío Bío, Ia Intendencia de la Región del Bío Bío y otros diversos organismos como la

4 Ver mapa en el anexo.

5 La comunidad de Malla Malla pertenece al grupo mapuche- pehuenche del Alto Bío Bío, quienes practican la actividad ganadera de trashumancia veranada-invernada para el pastoreo y recolección del pehuén o piñón desde las piñalerías de las veranadas que se ubican en las cumbres del cordón montañoso de la cordillera de los Andes.

6 Según se data en la escritura de compra del año 2001 en Temuco.

7 El origen del Municipio de Ralco es desde el Municipio de Santa Bárbara, con traslado de funcionarios e información del territorio. 
Iglesia Católica, no teniendo respuesta a sus demandas. Desde el análisis de las reuniones y los documentos extraídos, se observa que el Estado ha actuado en incumplimiento de su mandato y obligación, además de transgredir derechos fundamentales de este grupo de comuneros al denegar en su acción "el derecho al desarrollo" como lo es proclamado por el derecho internacional y las convenciones de Naciones Unidas. Cabe preguntarse por qué el Estado ha actuado en conocimiento de causa de una manera negligente, al no cumplir su mandato constitucional frente al pueblo mapuche.

Causas de la relocalización

Al momento del retorno de la democracia, siendo Ionco de Malla Malla Alberto Solar Huanque, se inicia un proceso de negociación con el Estado de Chile para la compra y recuperación del fundo Queuco, una extensión de tierras compuestas por valles, quebradas y veranadas de aproximadamente 15.000 has, que además es cruzado por el Rio Queuco, uno de los afluentes principales del río Bío Bío, y en cuyos cerros además, según fuentes de los propios comuneros y de las faenas ya realizadas en el fundo, existen importantes yacimientos mineros de cobre. Posteriormente esta demanda es llevada adelante desde 1996 frente a CONADI por J osé Martiniano Suárez en calidad de Ionco, momento en que el Estado de Chile por medio de CONADI comienza a negociar con las comunidades del Alto Bío Bío para enfrentar el difícil proceso de la construcción de la represa Ralco y la creciente organización de las comunidades indígenas en la Asociación de Loncos y el Consejo de Loncos.

Con la creciente organización de las comunidades indígenas, la intervención de organizaciones opositoras al gobierno de Chile, como el Consejo de Todas las Tierras, y la fuerte oposición de las comunidades a la construcción de la represa Ralco, es cuando el Estado de Chile comienza su intervención política por medio de CONADI y los organismos de seguridad como la Policía de Investigaciones, Carabineros y la Dirección de Seguridad Pública e Informaciones, DISPI, o "La Oficina" (Mella, 2007). De esta forma, además del ya histórico contexto de violencia generado en forma tradicional por el Estado de Chile, se agrega ahora la intervención política por los propios organismos de gestión de la política indígena y los organismos de inteligencia vigentes en la época. El objetivo de la intervención política aparecía con el objetivo de asegurar que no existiera un clima de subversión que afectara el control del territorio de parte del Estado, como a su vez no se pusiera en riesgo un proyecto considerado estratégico en materia energética por los gobiernos de la Concertación, como lo era la construcción de la represa Ralco. Es así como se aplican estrategias de empadronamiento, vigilancia, intervención política y contrainteligencia dentro de la estrategia de "sembrar cizaña" (Mella 2007, Aylwin y Yáñez, 2007). Esto último elevó el ya enrarecido clima en las comunidades, así como aumentó los niveles de desconfianza, división y violencia interna de las comunidades.

Por otro lado, la comunidad de Malla Malla, ya desesperanzada de la permanente negociación con CONADI, inicia la recuperación del fundo Queuco, asociados con comuneros de Cauñicu, encabezados por Agustina Huenupe Pavian y su hermano Mauricio Huenupe Pavian, existiendo oposición y desconfianza de parte de comuneros de Cauñicu, quienes no reparan en entrar en conflicto con los comuneros que demandaban el fundo Queuco apoyados por "colonos" y agentes del Estado en actividades de contrainteligencia. Es así que se construye en el valle del Queuco un clima de creciente violencia y desconfianza dentro de la demanda histórica de tierras y el surgimiento del movimiento indígena de recuperación de tierras en el Alto Bío Bío, el que sumado a la condición de pobreza va preparando situaciones de enfrentamiento y venganza entre los comuneros y "Ios colonos" y entre

8 A estas visitas y reuniones los investigadores fueron invitados en calidad de participantes como "testigos de fe" y se pudo realizar un registro fotográfico y audiovisual de ellas. 
Ios comuneros mismos. De esta forma se inicia la recuperación del fundo Queuco tanto por la comunidad de Malla Malla, encabezada esta vez por Segundo Suárez Marihuan, hermano de Pedro Segundo y de José Martiniano, y por un sector de la comunidad de Cauñicu encabezado por Agustina y Mauricio Huenupe Pavian.

El clima de violencia en el Alto Bío Bío en el contexto del movimiento de recuperación de tierras y de la construcción de la represa Ralco apuntaba a la generación casi espontánea de enfrentamientos, lo que se fue produciendo a pequeña escala hasta el año $2002^{9}$.

Es así como la intervención política del Estado, y de organizaciones opositoras al Estado de Chile, va creando un clima de división y enfrentamiento que termina por fragmentar las comunidades, situación que ya se había producido en el proceso de reduccionamiento por los Títulos de Merced, al reunir familias de diferentes líneas de parentesco que rivalizaban entre sí antes de la ocupación de su territorio.

De esta forma se crea, a nuestro entender, una tríada de factores relacionados entre sí que determinan los enfrentamientos directos ocurridos en julio de 2002 y que culminan con la muerte de Agustina Huenupe Pavian y de Mauricio Huenupe Pavian. Estos factores corresponden a: a) La situación de conflicto histórico no respondida por el Estado de Chile y que lleva a un clima de violencia permanente al interior de las comunidades, que se expresa en escasez de tierras, b) la intervención política por los organismos de seguridad del Estado de Chile, y c) la pobreza y la deteriorada calidad de vida que lleva a emigrar en forma espontánea y que no permite dar cabida a un desarrollo en un territorio escaso de recursos para la sobrevivencia. Así, la configuración de un clima de violencia, escasez de recursos, presión por nuevas tierras que permitan un desarrollo acorde a las necesidades culturales, la incapacidad del Estado de Chile por comprender las reales necesidades de las comunidades expresadas en políticas indígenas ineficientes y sesgadas, y la intervención política bajo la idea de la seguridad interior del Estado y de la tesis de la "subversión", Ileva a que el Estado de Chile transgreda principios fundamentales del derecho internacional sobre derechos humanos y genere en esta condición un clima de violencia que termina con los sucesos que finalmente motivaron el proceso de reasentamiento forzado de los comuneros en el sector de San Ramón-Michales formando la comunidad de Nehuen Mapu. Este suceso es la muerte de los hermanos Agustina y Mauricio Huenupe Pavian, delimitando un contexto claro de: a) violencia, b) pobreza crónica y c) intervención política del Estado de Chile.

\section{Análisis e interpretación del caso}

El caso de la comunidad de Nehuen Mapu refleja la consecuencia de cómo se aplican las políticas indígenas en Chile, la finalidad de la intervención de parte del Estado y los objetivos claros de los organismos de seguridad baj o el contexto de "criminalización" del movimiento indígena (Mella, 2007). La política indígena, que intentó una expresión de buena voluntad en la "Comisión de Verdad Histórica y Nuevo Trato" impulsada por el gobierno de turno, no constituye un medio de entendimiento y acercamiento que dé respuesta a las demandas sociales de los pueblos indígenas. En este caso se replican los mismos hechos que han marcado la relación histórica entre el Estado de Chile y los pueblos indígenas, que son de violencia estructural tanto externa como interna a la comunidad, una condición de pobreza extrema agravada por la falta de tierras para un desarrollo acorde a sus necesidades culturales, y la intervención política del Estado bajo el principio de control de la subversión y seguridad interior del Estado. Estas tres condiciones son detonantes de los procesos de enfrentamiento en las comunidades del Alto Bío Bío que lleva a la migración y la disolución de grupos familiares

9 Como relatan las fuentes periodísticas de la época 
que abandonan el territorio y su cultura formando parte de los grupos marginales de las ciudades y campos aledaños bajo una condición de extrema pobreza. Una situación que no tiene respuesta ni es solucionada por la actual política indígena. (Howe, 2005).

El abordaje original fue el estudio de la relación de la pobreza con la violación de derechos humanos, lo que llevó al planteamiento del modelo estructural; sin embargo, este modelo estaba incompleto en su función explicativa hasta poder establecer las reales condiciones del traslado y de la relocalización.

Propuesta de un modelo de evaluación de la política pública indígena

Los resultados de la investigación permitiría a UNESCO establecer un modelo que interprete los efectos de la intervención política, la violencia y las políticas públicas en las dinámicas sociales y culturales de la comunidad, como también a nivel de estructura interna- cognitiva de los individuos. Tales efectos podrían determinar decisiones y cambios en las comunidades indígenas, conduciéndolos a disolución de la comunidad en términos culturales.

El modelo propuesto aborda los elementos de intervención del Estado en las siete variables definidas como de acción externa, tales como la relación de territorialidad, acción política y efecto social Ilevado adelante por el Estado de Chile y la sociedad chilena. En las cinco variables dependientes es posible identificar también, de modo cuantitativo y cualitativo, los efectos visibles de la pobreza crónica y la violencia a que se ven sometidas las personas, familias y comunidades, estableciendo los patrones medibles de los efectos del cambio social en los pueblos indígenas y la relación directa con los indicadores de desarrollo. En tercer lugar, las variables endógenas muestran los efectos de la aplicación de las políticas públicas en la comunidad en términos de la relación político- económica, con efecto directo en el desarrollo de las comunidades. La variable de dinámicas internas establece los efectos que producen las otras variables en la estructura cognitiva de las personas y grupos étnicos que los orientan al desarrollo, esto es, capacidades, percepciones, conocimiento, organización, estructura social, identidad y dinámicas de cambio, pudiendo de esta forma postular una definición general de pobreza posible de aplicar en las políticas indígenas, en los términos siguientes:

"Pérdida de capacidades, seguridad y sentido de vida para construir desde ellos mismos su propio sistema de vida. Aquella pérdida es producto de daños inducidos por la acción de fuerzas externas, como guerras, desplazamiento involuntario, genocidio, hambrunas, cambio climático, contaminación, sequía, expropiación de tierras y acciones de control, dominio y ocupación del territorio, trayendo como consecuencia violencia, muerte, disolución de familias y estado de miedo permanente que afectan la organización interna de los pueblos indígenas".

\section{Discusión: análisis de las dinámicas internas}

Las manifestaciones de estos procesos de intervención política y violencia pueden ser interpretados desde las estructuras cognitivas de los grupos intervenidos, una interpretación que aborda las dinámicas internas de las comunidades en su nivel de relaciones sociales, políticas y culturales que surgen desde la estructura familiar y de parentesco. La relación política interna de las comunidades indígenas se organiza desde los lazos establecidos por la estructura política del Lof como unidad sociopolítica básica que emerge desde la organización familiar. Los estudios de la construcción familiar desde los nexos que emergen desde el linaje muestran como base de organización una estratificación social que ordena las dinámicas familiares (Durán y Catriquir, 2007), las que por los procesos de intervención son alteradas y colocadas en conflicto. La disputa interna por efecto de la pobreza se ve más agravada cuando el Estado 
interviene con planes de superación de pobreza estableciendo las redes de clientelismo y asistencialidad que sobrepasa las estructuras familiares internas. La organización familiar y la organización sociopolítica se alteran en razón a que los patrones de autoridad en el sentido de los códigos políticos y religiosos ya no son valorados como fuentes de seguridad y recursos para el equilibrio de la comunidad.

Una de las situaciones observadas en los procesos de reasentamiento dirigido desde la política de CONADI es que no guardan pertinencia cultural con los grupos relocalizados; ello, al no conservar la organización familiar, social y política del conjunto de personas trasladadas (Aylwin y Yáñez 2007). Lo anterior genera tensiones al interior de los grupos familiares y comunidades y se presta para divisiones que son utilizadas por la intervención política de diversos grupos, trayendo en sí nuevas fuentes de conflictos intracomunitarios que se traducen en problemas de violencia al interior de las comunidades.

La falta de un enfoque orientado a reconocer la organización familiar tradicional como núcleo básico en lo social, político, económico y cultural es parte de la carencia de claridad en la legislación nacional, donde la Ley 19.253 y la política de Nuevo Trato reconoce a las comunidades legalmente constituidas pero no a la organización sociopolítica tradicional que agrupa a los núcleos familiares, siendo este el Lof con su agrupación de Lof Che y Lof Mapu, que a su vez contiene a los Rucache y los Reñma, englobados a su vez, por el factor Küga o "Iinaje" (Quidel et al. 2007). Las organizaciones tradicionales no son solo una expresión de costumbres y "folclore", constituyen un medio de expresión de organización social en torno a un territorio, así como una expresión de la organización de estatus dentro de la propia sociedad mapuche.

Este tipo de organización sociopolítica mapuche es la que se ve alterada en los procesos de desplazamiento, relocalización y reasentamientos, lo que en definitiva no significa solo una alteración de agrupaciones familiares, sino de condiciones propias del desarrollo económico, social, cultural y político de la población involucrada. Al estar unida la organización familiar a un territorio, y derivando esta organización familiar en una expresión política y económica, el resultado de las alteraciones socio familiares se traduce en condiciones de pobreza y de deterioro de desarrollo humano, como a su vez una transgresión al derecho al desarrollo y también los derechos políticos y culturales del pueblo mapuche (Durán y Catriquir, 2007). Así, la falta del reconocimiento de esta expresión sociopolítica y de la insuficiencia de un marco constitucional que las legitime, sea a través del Convenio 169 o de su reconocimiento constitucional, implica que en sí la política indígena y la Ley 19.253 no tengan el efecto esperado de desarrollo de las comunidades indígenas, ni en este caso del pueblo mapuche, en específico.

En el caso de las comunidades pehuenches, debido a los procesos de relocalización, desplazamiento y exterminio por efecto de la colonización de sus territorios, como a su vez la posterior ocupación por efecto de los megaproyectos, se puede observar que esta agrupación de Lof Che y Lof Mapu se reduce a en parcelaciones pequeñas que no generan un autosustento familiar; significando pobreza y disolución de núcleos familiares, como a su vez estados internos de violencia. Estas agrupaciones en el Alto Bío Bío se conocen como "caserados" y corresponderían a la misma lógica del Lof Che y Lof Mapu, solo que sin relación a la posesión de un territorio que les permita un desarrollo y reproducción sociocultural bajo los patrones de sus normas internas. Consideramos que por efecto de las políticas indígenas y de desarrollo propiciado por el marco legislativo vigente es que no se hace posible resolver las situaciones de conflicto interno y de pobreza de las comunidades indígenas. Por otro lado, la respuesta política de las comunidades es difusa para los organismos de estado ya que no están bajo los patrones de "organizaciones legalmente constituidas" que permita una coherencia en la interpretación de sus 
demandas. El esquema político del Estado es muy diferente al esquema político cultural de las organizaciones políticas mapuche, lo que se traduce en una fuerte tensión, violencia interétnica y violencia política.

De esta manera, la asociación entre la expresión política tradicional y los medios legales establecidos por el estado de derecho es dispar y disonante. Por otro lado, los marcos institucionales que permiten un mejor entendimiento y acercamiento entre las partes involucradas no son considerados aún por el propio Estado y el reconocimiento constitucional. Como se sabe, en estos casos se aplica el concepto de pueblo que de ningún modo implica una separación del Estado, sino supone una condición de reconocimiento a la diversidad dentro de un Estado y de una nación que aborda la representatividad y derechos políticos, ciudadanos, y económicos, sociales y culturales, desde la condición pluriétnica - multiétnica dentro de una pluralidad cultural. La otra ventaja de estos esquemas internacionales es que permiten abordar con mayor propiedad los problemas de desarrollo local y de la distribución de la riqueza, como de la misma manera un esquema más definido para la formulación, planificación, gestión y aplicación de las políticas públicas vigentes para el desarrollo particular del tema indígena.

Las actividades ligadas a un territorio fundamentalmente son de tipo económico; en el caso del territorio y los grupos humanos que lo pueblan, estas actividades pueden dividirse en actividades agrícolas o ganaderas, siendo en estos casos de pequeña agricultura y de pastoreo. Así es posible dividir los grupos de comunidades indígenas según el principal tipo de actividad económica que determina una característica de asociación y desarrollo. En el caso de las comunidades del sector valle central su actividad económica está asociada a una pequeña agricultura y actividades de pastoreo, hoy complementadas con actividades forestales. En el caso de las comunidades de la costa hay un complemento con actividades de pesca y recolección, y en las comunidades de Alto Bío Bío hay una actividad asociada a pastoreo de pequeña escala que es fuertemente afectada por los factores climáticos. De esta forma es posible esperar especializaciones productivas en algunas comunidades que determinan las organizaciones sociales y políticas, como es el caso de las comunidades del alto Bio Bio con la recolección del piñón y el pastoreo de veranada.

Las actividades productivas deben ser consideradas en las actividades de relocalización y reasentamiento según nivel de especialización productiva, en el marco de una economía de subsistencia familiar como lo es la recolección del piñón. También lo es en este tipo de actividad productiva la organización familiar que se define en torno a ella, sean "caserados", Lof Che y Lof Mapu, ya que va a orientar las formas de relocalización y reasentamiento. Asimismo, estas agrupaciones familiares y sociales que se estructuran además de la relación de parentesco a la organización sociopolítica, son las que estructuran las relaciones de cooperación en las actividades productivas, sea para explotación de los recursos desde la perspectiva agrícola 0 ganadera, o para actividades de trabaj o fuera de la comunidad, como lo es la subcontratación forestal, hortofrutícola, de construcción, e incluso de labores domésticas. Así, la actividad económica del grupo familiar no es de ninguna manera una actividad aislada y realizada en forma individual, sino que sigue los patrones de asociatividad establecidos por las redes de cooperación desde el Reñma, Rucache $y$ Lof Che, donde incluso en los sectores urbanos aún priman los códigos de ética y de comportamiento de las comunidades originales (op. cit., 2007). Estas dinámicas internas constituyen así una especialización en la construcción del bienestar de los pueblos indígenas acordes a un principio de cultura y organización de su estructura social.

\section{Conclusiones}

Se extrae tanto del estudio de caso como de la investigación para UNESCO que las condiciones que generan la ausencia de 
condiciones de desarrollo corresponden a los elementos de violencia y pobreza crónica que generan los cambios sociales que determinan los procesos de disolución cultural y deterioro, de comunidades indígenas en América Latina, como a su vez estas condiciones llevan a un clima de relaciones políticas que se sustentan en el conflicto y que el Estado de Chile actúa de una manera equivocada para dar respuesta a ella. La respuesta de intervención política, orientada sobre la tesis de seguridad interior del estado, y de políticas públicas paternalistas, determinan un curso de relaciones que potencian el conflicto y generan un clima de violencia social proveniente del Estado de Chile que llevó a los sucesos que se detallaron anteriormente. Se entiende de esta manera que el tipo de desarrollo de las comunidades indígenas se inscribe en todas las variables asociadas a la pobreza, la falta de tierras, el asistencialismo, el clientelismo y la intervención política, que llevan a lo señalado por dependencia, escasez de recursos y pérdida de capacidades. En esta situación de carencia de desarrollo es posible identificar tres variables que marcan la vida y relación de las comunidades con el Estado de Chile, que son a) Violencia, b) Pobreza Crónica, y c) Intervención Política.

Así, en la pobreza crónica se identifica el modelo estructural que muestra una carencia de las políticas públicas indígenas en relación a criterios de pertinencia cultural. La condición de pobreza que se homologa a una falta de desarrollo demostraría que la relación entre el Estado de Chile con los pueblos indígenas no se establecería con pertinencia cuando la situación de exclusión, persecución y las demandas históricas de tierras no son restituidas de una manera acorde a su cultura. Por otro lado, las demandas indígenas por territorio, autonomía y reconocimiento, al no tener respuesta a nivel constitucional por el Estado de Chile, impiden generar políticas públicas adecuadas para enfrentar el problema de la pobreza.

El artículo trabaja también la idea de que la tesis de la seguridad interior del Estado y la actitud paternalista no permiten generar un marco adecuado de relaciones sociopolíticas acorde a las demandas de distintos sectores del pueblo mapuche.

\section{BIBLIOGRAFÍA}

AYLWIN, J y N. YÁÑEZ. (2007), El Gobierno de Lagos, los pueblos indígenas y el "nuevo trato". Las paradojas de la democracia chilena. LOM y Observatorio Derechos Indígenas de Pueblos Indígenas. Santiago de Chile.

COLECTIVO DE DETENIDOS PEHUENCHES DE LA COMUNIDAD DE CAUÑICÚ. (2006), "Cartas Pehuenches 2002-2005". En Anales de Desclasificación Vol.1 La derrota del área cultural $N^{\circ} 2$. Santiago de Chile.

DURÁN, T y J. QUIDEL (2007), “Reducción y comunidad. Visión externa al lof che y al lof mapu". En Patrimonio Cultural Mapunche. Derechos Sociales y Patrimonio Institucional Mapunche, Volumen III. Temuco.

DURÁN, T y D. CATRIQUIR (2007), “Mapun üy: El nombre personal en la sociedad y cultura mapunche. Implicancias étnicas y sociales". En Patrimonio Cultural Mapunche. Derechos Sociales y Patrimonio Institucional Mapunche, Volumen III. Temuco.

GOBIERNO DE CHILE (2004), "Política de Nuevo Trato con los Pueblos Indígenas; Derechos Indígenas, Desarrollo con Identidad y Diversidad Cultural". Santiago de Chile.

GOBIERNO DE CHILE (2004), “Intervención del Presidente de la República, Ricardo Lagos, durante la ceremonia de entrega de políticas de Nuevo Trato a los Pueblos Indígenas 20042010". Santiago de Chile.

HOWE, G y A. MACKAY (2005), "Combining Quantitative and Qualitative methods in Assessing Chronic Poverty. The Case of Rwanda". En Q-Squared Working Paper $N^{\circ} 3$. Cornell University. U.S.A.

HULME, D. (2007), “Integrating Qualitative and Quantitative Research for Country Case 


\section{UNIVERSIDAD CATÓLICA DE TEMUCO}

Studies of Development". En Global Poverty Research Group and Economic and Social Research Council, University of Manchester. United Kingdom.

MELLA, E. (2007), Los mapuche ante la justicia. La criminalización de la protesta indígena en Chile. LOM y Observatorio Derechos de Pueblos Indígenas. Santiago de Chile.

NACIONES UNIDAS, CONSEJO ECONÓMICO Y SOCIAL, COMISIÓN DE DERECHOS HUMANOS (2005), Las Cuestiones Indígenas, los derechos humanos y las cuestiones indígenas, Informe del Relator Especial sobre la situación de los derechos humanos y las libertades fundamentales de los indígenas Rodolfo Stavenhagen. Nueva York.

OIT (1989), "Convenio sobre pueblos indígenas en Países Independientes (Convenio 169)". Ginebra Suiza.

QUIDEL J. T. DURAN y D. CATRIQUIR (2007), "Rukache o Familia Mapunche: La relación entre Reñma y Mapu". Patrimonio Cultural Mapunche. Derechos Sociales y Patrimonio Institucional Mapunche, Volumen III, pag 395414. 
$N^{\circ}$ 1. Mapa de Comunidad Nehuen Mapu.

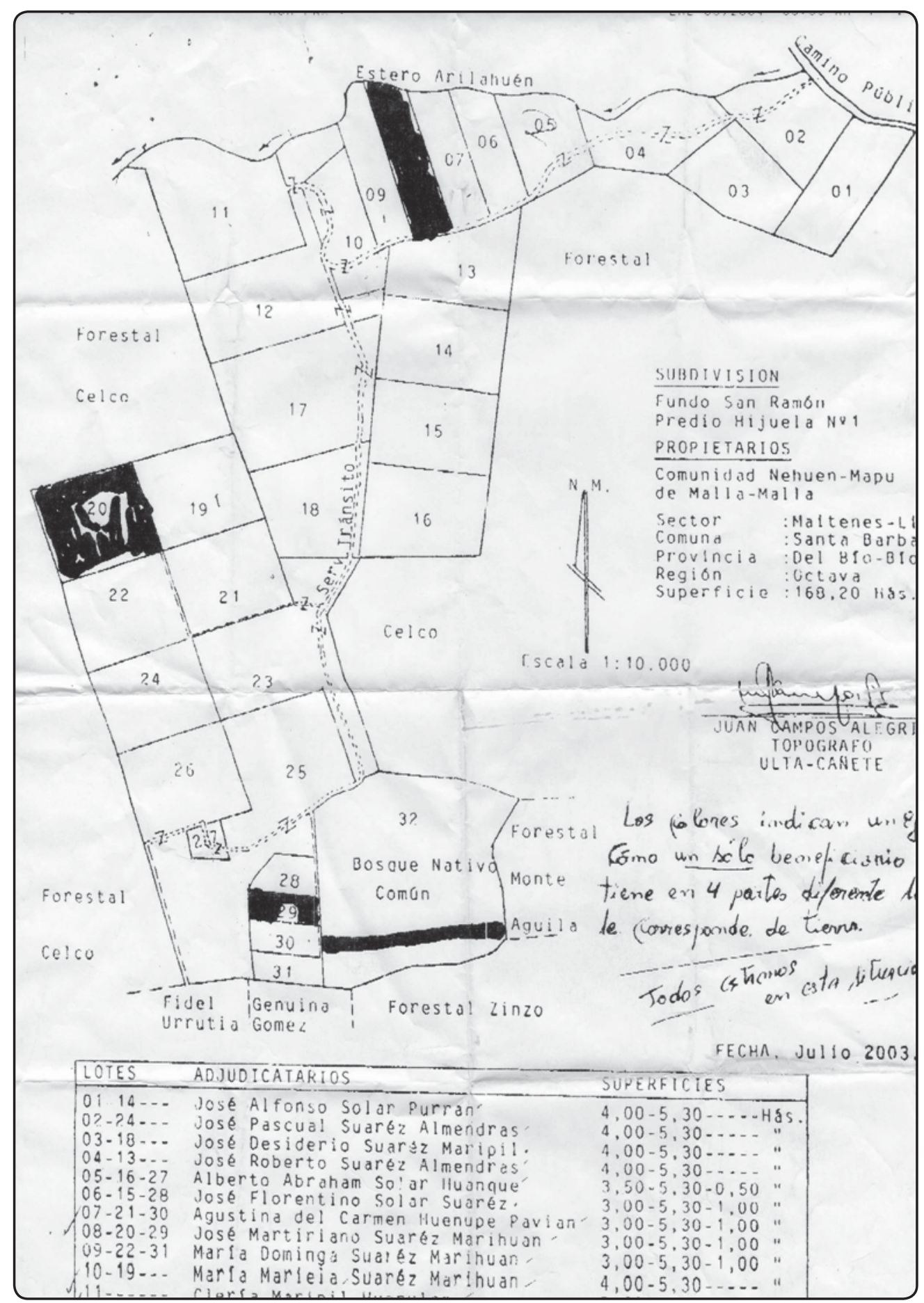


$N^{\circ}$ 2. Modelo Estructural. (Pacheco et al.)

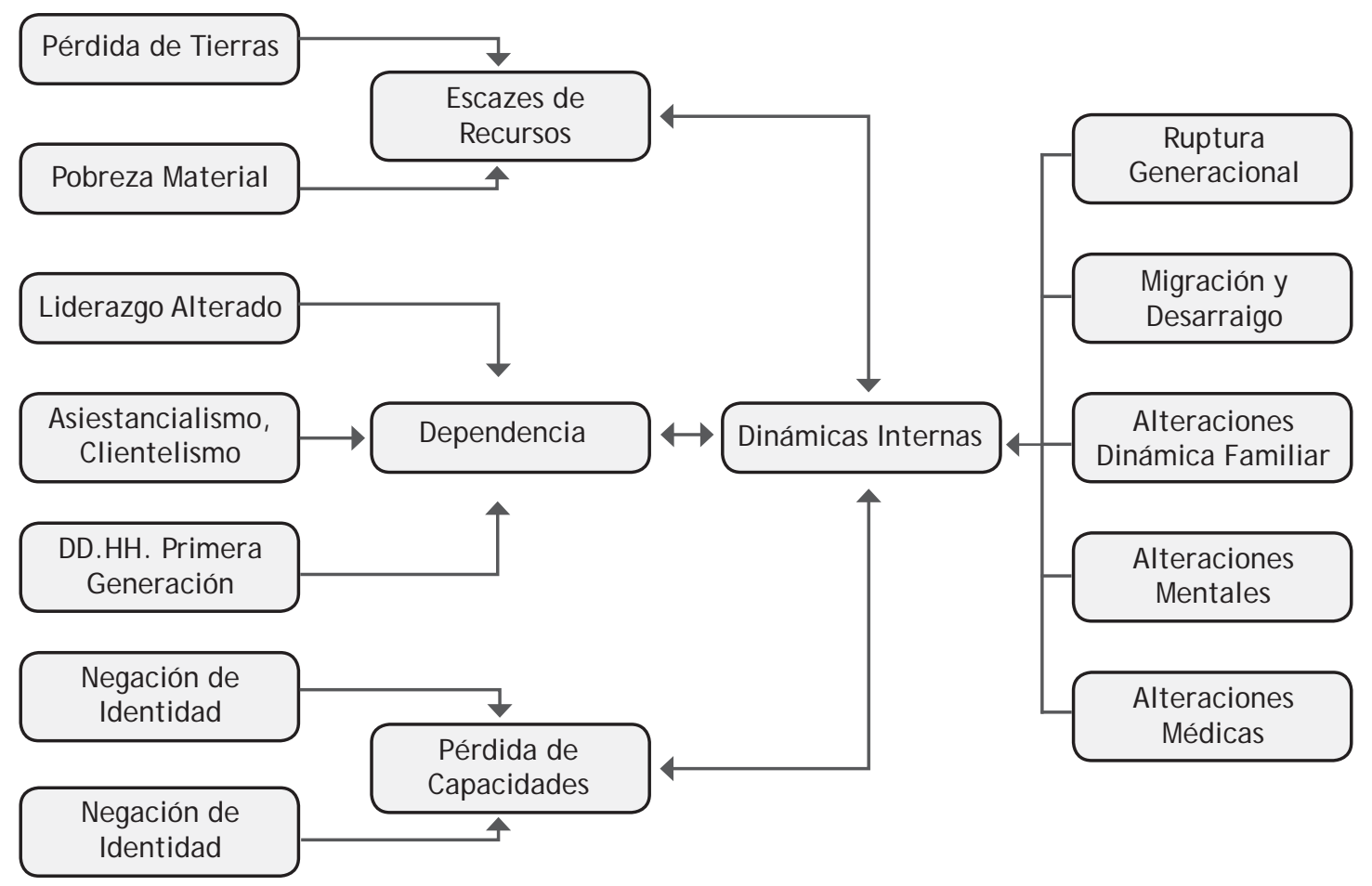

